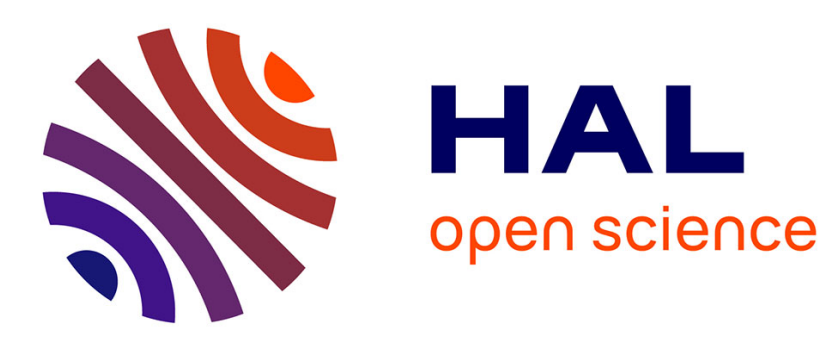

\title{
Interplay between Local Anisotropies in Binuclear Complexes
}

\author{
Renaud Ruamps, Rémi Maurice, Coen de Graaf, Nathalie Guihéry
}

\section{To cite this version:}

Renaud Ruamps, Rémi Maurice, Coen de Graaf, Nathalie Guihéry. Interplay between Local Anisotropies in Binuclear Complexes. Inorganic Chemistry, 2014, 53, pp.4508-4516. 10.1021/ic500180k . in2p3-00983068

\section{HAL Id: in2p3-00983068 https://hal.in2p3.fr/in2p3-00983068}

Submitted on 24 Apr 2014

HAL is a multi-disciplinary open access archive for the deposit and dissemination of scientific research documents, whether they are published or not. The documents may come from teaching and research institutions in France or abroad, or from public or private research centers.
L'archive ouverte pluridisciplinaire HAL, est destinée au dépôt et à la diffusion de documents scientifiques de niveau recherche, publiés ou non, émanant des établissements d'enseignement et de recherche français ou étrangers, des laboratoires publics ou privés. 


\title{
Interplay between local anisotropies in binuclear complexes
}

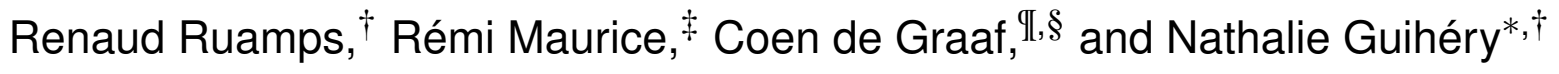 \\ Laboratoire de Chimie et de Physique Quantiques, IRSAMC/UMR5626, Université de Toulouse 3, \\ 118 route de Narbonne, F-31062 Toulouse Cédex 4, France, SUBATECH, UMR CNRS 6457, \\ IN2P3/EMN Nantes/Université de Nantes, 4 rue Alfred Kastler, BP 20722, 44307 Nantes Cédex \\ 3, France, Departament de Química Física i Inorgànica, Universitat Rovira i Virgili, Marcel-lí \\ Domingo s/n, 43007 Tarragona, Spain, and Institució Catalana de Recerca i Estudis Avançats \\ (ICREA), Passeig Lluís Companys 23, 08010, Barcelona, Spain \\ E-mail: nathalie.guihery@irsamc.ups-tlse.fr
}

April 1, 2014

\footnotetext{
* To whom correspondence should be addressed

†Université de Toulouse

†IN2P3/EMN Nantes/Université de Nantes

IUniversitat Rovira i Virgili

$\S_{\text {ICREA }}$
} 


\begin{abstract}
A systematic study has been undertaken to determine how local distortions affect the overall (molecular) magnetic anisotropies in binuclear complexes. For this purpose we have applied a series of distortions to two binuclear Ni(II) model complexes and extracted the magnetic anisotropy parameters of multispin and giant-spin model Hamiltonians. Furthermore local and molecular magnetic axes frames have been determined. It is shown that certain combinations of local distortions can lead to constructive interference of the local anisotropies and that the largest contribution to the anisotropic exchange does not arise from the second-rank tensor normally included in the multispin Hamiltonian, but rather from a fourth-rank tensor. From the comparison of the extracted parameters, simple rules are obtained to maximize the molecular anisotropy by controlling the local magnetic anisotropy, which opens the way to tune the anisotropy in binuclear or polynuclear complexes.
\end{abstract}




\section{Introduction}

Magnetic anisotropy is the origin of the single molecule magnet (SMM) behavior ${ }^{1-5}$ which consists in a slow relaxation of the magnetization, and a blocking of the magnetization for low enough temperatures. Since this bistable behavior may lead to technological applications in the domain of data storage ${ }^{6}$ and quantum computing, ${ }^{7-9}$ the understanding of the microscopic origin of magnetic anisotropy has received considerable interest during the last two decades. For most of the transition metal (TM) complexes, the property arises from the loss of degeneracy of the $M_{S}$ components of the ground spin state $S$ due to relativistic effects, in particular the spin-orbit coupling, combined with geometrical distortions from the highly symmetric octahedral or tetrahedral situations. This phenomenon is called the Zero-Field Splitting (ZFS) and is characterized by the axial $D$ and the rhombic $E$ parameters. Magnetic anisotropy can also be observed in cases where the angular momentum is not quenched in the pure electronic ground state, due to for instance molecular orbital (near-)degeneracy in TM complexes, and lanthanide or actinide complexes. In such cases a pseudo-spin $\tilde{S}$ can be defined which may significantly differ from the true spin state. ${ }^{10}$

For an even number of unpaired electrons, bistable behavior occurs when the complex has a uniaxial magnetic anisotropy and the two maximal $\left|M_{S}\right|$ components of the ground spin state are the lowest degenerate states, i.e. when $E=0$ and $D$ is large and negative. In case of non-zero rhombic anisotropy $(E \neq 0)$, the $M_{S}^{\min }$ and $M_{S}^{\max }$ components are coupled and the ground state is a linear combination of $M_{S}$ components. When $D$ is positive, the lowest $\left|M_{S}\right|$ component becomes the dominant component of the ground state and no bistability can be observed. In the case of an odd number of unpaired electrons, slow relaxation of the magnetization can a priori be observed even if the ground Kramers doublet is essentially composed of the lowest $\left|M_{S}\right|$ components (corresponding to a positive $D$ value), and even if the rhombic parameter $E$ does not vanish. ${ }^{11}$ Nevertheless, such systems are not very common and also for odd-electron systems negative $D$-values are central to SMM behavior. The synthesis of new objects with improved anisotropy characteristics rests on the ability to control the nature (uniaxial or in plane) and the magnitude of the magnetic anisotropy. Several theoretical works have been devoted in the last years to the understanding of the chemical 
and geometrical factors that govern the magnetic anisotropy in mononuclear species. ${ }^{12-17}$ While due to the numerous driving forces (configurations $\mathrm{d}^{n}$, geometries, strength of the ligand field, etc.) the conclusions of these studies are quite often system-specific, theory allows one to understand and accurately predict the magnetic anisotropy characteristics of mononuclear species. In polynuclear complexes, the situation is more complicated since additional factors are expected to come into play:

- the different ions in the complex are likely to have different local anisotropies. Indeed, the relativistic effects, responsible for the loss of degeneracy of the spin components of the ground spin state $S_{i}$ of each ion $i$, are essentially local and hence, very sensitive to the local environment of each ion, which may significantly differ from ion to ion.

- intersite anisotropic interactions (such as the anisotropic exchange) may be present, affecting the characteristics of the overall magnetic anisotropy of the polynuclear complex, here called the molecular anisotropy.

The main aim of the present study is to advance in the understanding of synergistic effects between local anisotropies. We consider model binuclear complexes constituted of two Ni(II) ions adopting various geometries for which both the nature and the magnitude of the local anisotropies may or may not be different. In all the cases considered, the parameters characterizing the local anisotropies are determined and confronted to those of the molecular magnetic anisotropy of the complex. Two types of anisotropic spin Hamiltonians can be used to characterize the ZFS of polynuclear complexes:

- The giant-spin Hamiltonian reproduces the energy levels of a single spin state, usually the ground spin state. Nevertheless several giant-spin Hamiltonians can be extracted to describe the energy levels of all the different spin states of a complex. When the molecular system contains four unpaired electrons or more $(S \geq 2)$, and in particular in the weak-exchange limit, i.e. when the mixing between the ground and the excited spin states is non-negligible, this model Hamiltonian contains spin operators of higher order than two. 
- The multispin Hamiltonian reproduces the energy of the $M_{S}$ components of all spin states arising from the coupling between the local spin states of the paramagnetic ions. This Hamiltonian is spanned in the uncoupled $\left|S_{a}, M_{S_{a}}, S_{b}, M_{S_{b}}\right\rangle$ basis and accounts for the spin mixing.

In recent studies, the physical content of these two Hamiltonians has been confronted to ab initio calculations based on the all-electron Hamiltonian. ${ }^{18,19}$ It was shown that the usual approximations made in these models are not suitable to reproduce the interactions resulting from the ab initio calculations of a binuclear $\mathrm{Ni}(\mathrm{II})$ complex in the weak-exchange limit. In the case of the giant-spin Hamiltonian, simple additional operators were sufficient to consistently introduce the spin-mixing effect on the effective splitting and mixing of the $M_{S}$ components of the ground spin state, and all non-negligible interactions could be extracted. ${ }^{19}$ Concerning the multispin Hamiltonian, it was shown that a biquadratic operator and a four-rank tensor should be introduced to reproduce accurately all the effective non-negligible interactions arising from the all-electron Hamiltonian. ${ }^{18}$ Unfortunately, the number of these interactions was too large and a full extraction could not be performed. Owing to recent advances in our extraction procedure, such an extraction is now possible and an important issue of the present paper is to determine all non-negligible anisotropic interactions. Quite surprisingly, it will be shown that the fourth-rank tensor actually brings the main contributions to the exchange anisotropy.

It should be stressed that relations between the parameters of the local and molecular anisotropy tensors already exist, ${ }^{20-23}$ and that it is actually possible to determine the molecular magnetic anisotropy tensors by combining the local ones if two hypotheses are made, (i) the anisotropy axes on both magnetic centers are parallel, and (ii) the anisotropic intersite interactions of the two centers are negligible. The present paper quantifies and discusses the various anisotropic interactions including those of the fourth-rank symmetric tensor from $a b$ initio calculations, allowing us to determine the local and molecular anisotropies without the necessity of the mentioned assumptions.

The next section briefly presents the procedure of extraction of the model interactions from the effective Hamiltonian theory and provides the computational information. Section III recalls the physics of the two considered spin Hamiltonians and presents a method for the determination of the 
molecular and local magnetic axes frames. Section IV discusses the magnitude and nature of the extracted local and molecular anisotropic interactions and analyses interference effects between local anisotropies on the molecular anisotropy.

\section{Method of extraction and computational information}

As shown in previous studies, ${ }^{24-27}$ it is possible to establish the relevance of any model Hamiltonian and to extract its constitutive interactions by using the effective Hamiltonian theory. In combination with correlated $a b$ initio calculations performed using the all-electron Hamiltonian, the effective Hamiltonian theory enables one to numerically evaluate all the matrix elements of a model Hamiltonian. This method has successfully been applied to mono- and bi-nuclear systems to determine anisotropic interactions of the giant-spin and multispin Hamiltonians. ${ }^{13,18,19,28-31}$

The effective Hamiltonian theory ${ }^{32,33}$ enables one to extract from accurate $a b$ initio calculations the most rigorous effective Hamiltonian working in the same model space as the model Hamiltonian. This effective Hamiltonian is then compared to the model one. In the des Cloizeaux formalism, ${ }^{33}$ the general expression of the effective Hamiltonian is:

$$
\hat{H}^{e f f}=\sum_{k}\left|\tilde{\Psi}_{k}\right\rangle E_{k}\left\langle\tilde{\Psi}_{k}\right|
$$

where $\tilde{\Psi}_{k}$ are the symmetrically orthogonalized and normalized projections onto the model space of the all-electron Hamiltonian eigenvectors $\Psi_{k}$, and $E_{k}$ are the corresponding eigenvalues. This formulation ensures that the eigenvalues of the effective Hamiltonian are the energies of the allelectron Hamiltonian, while the eigenvectors of the effective Hamiltonian are the projections onto the model space of the all-electron Hamiltonian eigenvectors, such that:

$$
\hat{H}^{e f f}\left|\tilde{\Psi}_{k}\right\rangle=E_{k}\left|\tilde{\Psi}_{k}\right\rangle
$$


Since it is possible to calculate all the matrix elements of the effective Hamiltonian as:

$$
\left\langle i\left|\hat{H}^{e f f}\right| j\right\rangle=\left\langle i\left|\sum_{k}\right| \tilde{\Psi}_{k}\right\rangle E_{k}\left\langle\tilde{\Psi}_{k} \mid j\right\rangle
$$

the method provides more information than the low energy spectrum. Values of the interactions of the model Hamiltonian can be assigned by confronting these numerical matrix elements to their analytical expression in the model Hamiltonian.

The ab initio calculations were performed using the Spin-Orbit State-Interaction (SO-SI) method ${ }^{34,35}$ implemented in the MOLCAS package. ${ }^{36,37}$ The method performs a variational treatment of the spin-orbit couplings between the lowest selected states. The preliminary spin-orbit free calculations account for non-dynamic correlation effects through the complete active space self-consistent field (CASSCF) method. The active space contains the $16 \mathrm{~d}$ electrons in the $10 \mathrm{~d}$ orbitals for the calculation of the magnetic anisotropy of the $\mathrm{Ni}$ (II) binuclear species, i.e. CAS(16,10)SCF. To compute the local anisotropy tensors, the orbitals of each center were considered active alternatively while the orbitals of the other center were kept inactive, i.e. CAS $(8,5) \mathrm{SCF}$ calculations were carried out. Extended basis sets of ANO type ${ }^{38,39}$ have been used with the following contractions: 6s5p4d2f for $\mathrm{Ni}, 6 \mathrm{~s} 5 \mathrm{p} 2 \mathrm{~d}$ for $\mathrm{Cl}, 4 \mathrm{~s} 3 \mathrm{p} 1 \mathrm{~d}$ for $\mathrm{O}$ and $\mathrm{N}, 3 \mathrm{~s} 2 \mathrm{p}$ for $\mathrm{C}$ and $2 \mathrm{~s}$ for $\mathrm{H}$.

\section{Model Hamiltonians and the magnetic axes frame}

\subsection{Giant-Spin Hamiltonians and Molecular Anisotropy Tensors}

The simplest description of magnetic anisotropy in polynuclear systems is provided by the giantspin approximation. ${ }^{40-43}$ The use of this Hamiltonian is physically justified when the spin ground state of the molecule is sufficiently separated in energy from the other spin multiplets such that the magnetic properties can be described using a single spin ground state. Simultaneously, the ZFS of

other spin multiplets can be independently described, which leads to a block-spin Hamiltonian if all the coupled spin states and the isotropic couplings are considered. In this work, we will focus 
in particular on the $D$ and $E$ parameters of the triplet and quintet states, referred to as $D_{1}, E_{1}, D_{2}$ and $E_{2}$, respectively.

The giant-spin Hamiltonian can be expressed in terms the standard Stevens equivalent operators $^{44}$ and additional operators which were shown to be necessary when $S=2$ in the case of a binuclear complex in the weak-exchange limit. ${ }^{19}$ Nevertheless, in case of a strong exchange coupling between the magnetic ions, ${ }^{13}$ it can be reduced to its simple form:

$$
\hat{H}_{G S H}=\hat{S} \overline{\bar{D}} \hat{S}
$$

where $\hat{S}$ is the spin operator of the state under consideration which in our case will be either the triplet $(S=1)$ or the quintet $(S=2)$ state for which the second-rank associated tensors will be denoted $\overline{\bar{D}}_{1}$ and $\overline{\bar{D}}_{2}$, respectively.

Higher than second order terms become particularly significant when couplings between the different spin states (i.e. spin-mixing) are important, but are small in the here-considered cases since the isotropic magnetic coupling is relatively large (around $40 \mathrm{~cm}^{-1}$ ), i.e. we are in a situation close to the strong-exchange limit. The values of these interactions are not reported here since the largest value obtained is $0.09 \mathrm{~cm}^{-1}$, affecting the $\left\langle 2, \pm 2\left|\hat{H}^{e f f}\right| 2, \mp 2\right\rangle$ matrix elements by not more than $\sim 1 \mathrm{~cm}^{-1}$. The determination of the magnetic axes frame could therefore safely be performed using only the second-order tensors $S \cdot \overline{\bar{D}}_{2} \cdot S$ for the quintet state and $S \cdot \overline{\bar{D}}_{1} \cdot S$ for the triplet state (i.e. only the $B_{2}^{0}$ and $B_{2}^{2} \mathrm{D}_{2}, \mathrm{E}_{2}$ and $\mathrm{D}_{1}, \mathrm{E}_{1}$ parameters for both spin blocks are extracted). For this purpose, we have artificially removed the couplings between the singlet, triplet and quintet $M_{S}$ components to extract the block spin anisotropy tensors and consequently diagonalize them to find the anisotropy axes, as proposed in a previous work. ${ }^{19}$

\subsection{Multispin Hamiltonian}

For a binuclear complex constituted of sites $a$ and $b$, the multispin Hamiltonian works on the basis of the uncoupled $\left|M_{S_{a}}, M_{S_{b}}\right\rangle$ functions. It is designed to reproduce the energy of all the states 
resulting from the coupling between the ground spin states of each magnetic site. In the present study, the Hamiltonian describes the energy of all $M_{S}$ components of the singlet, triplet and quintet states after transformation to the coupled $\left|S, M_{S}\right\rangle$ basis. As recently shown in a binuclear complex of $\mathrm{Ni}(\mathrm{II})$ in the weak-exchange limit, it involves biquadratic operators and a fourth-rank tensor $\overline{\bar{D}}_{a a b b}$. In the considered case, its expression is:

$$
\hat{H}^{M S}=J \hat{S}_{a} \cdot \hat{S}_{b}+\hat{S}_{a} \overline{\bar{D}}_{a} \hat{S}_{a}+\hat{S}_{b} \overline{\bar{D}}_{b} \hat{S}_{b}+\hat{S}_{a} \overline{\bar{D}}_{a b} \hat{S}_{b}+\bar{d}_{a b} \hat{S}_{a} \times \hat{S}_{b}+\left(\hat{S}_{a} \otimes \hat{S}_{a}\right) \overline{\overline{\bar{D}}}_{a a b b}\left(\hat{S}_{b} \otimes \hat{S}_{b}\right)
$$

where $J$ is the isotropic magnetic exchange, $\overline{\bar{D}}_{a}$ and $\overline{\bar{D}}_{b}$ are local tensors, $\overline{\bar{D}}_{a b}$ is the symmetric anisotropic exchange tensor and $\bar{d}_{a b}$ is the antisymmetric anisotropic term, ${ }^{20-23}$ known as the Dzyaloshinskii Moriya pseudo-vector. The components of these two tensors read:

$$
=\left(\begin{array}{ccc}
\bar{D}_{a b} & +\bar{d}_{a b} \\
D^{x x} & D^{x y} & D^{x z} \\
D^{x y} & D^{y y} & D^{y z} \\
D^{x z} & D^{y z} & D^{z z}
\end{array}\right)+\left(\begin{array}{ccc}
0 & d^{x y} & -d^{x z} \\
-d^{x y} & 0 & d^{y z} \\
d^{x z} & -d^{y z} & 0
\end{array}\right)
$$

Note that the analytical Hamiltonian matrix is given in Ref. 18 for centrosymmetric cases.

To compare the nature of the local and molecular anisotropies, the magnetic anisotropy axes have been determined for each $\mathrm{Ni}(\mathrm{II})$ ions of the binuclear system in its triplet and quintet coupled spin states. For this purpose, the parameters of the various tensors of both Hamiltonians giant-spin and multispin were extracted from the effective Hamiltonian theory, such that the model Hamiltonian matrix calculated using the extracted values of the parameters reproduces at best the numerical effective Hamiltonian matrix calculated using equation 3.

Because the proper magnetic axes of each tensor may be different, all tensors are computed in a single axes frame and rotations $\left(P=R_{z}(\phi) \cdot R_{x}(\theta) \cdot R_{z}(\psi)\right.$ where $R_{x}$ and $R_{z}$ stand for rotation around $x$ and $z$ respectively and $\phi, \theta, \psi$ are the Euler angles) between the proper axes frame of each tensor and the axes frame of the calculation are introduced, enabling one to determine the 
axial and rhombic parameters of all magnetic anisotropy tensors. Tables 1 and 2 give the number and nature of the non-zero parameters depending on the symmetry point group for the second-rank tensor $\overline{\bar{D}}_{a b}$, the Dzyaloshinski Moriya vector and the fourth-rank tensor. $\kappa= \pm 1$ indicates the absence/presence of a symmetry element that interchanges the two magnetic centers. In order to simplify the extraction, we have imposed the following relation:

$$
D^{x x x x}+D^{y y y y}+D^{z z z z}+2 D^{x x y y}+2 D^{x x z z}+2 D^{y y z z}=0
$$

which has no other effect than ensuring that the anisotropic part of the Hamiltonian is traceless. To further reduce the number of independent variables, we make use of the fact that several matrix elements in the numerical effective Hamiltonian are (nearly) zero, whereas the corresponding elements of the model Hamiltonian is a (sum of) parameter(s). This introduces the following additional relations:

$$
\begin{aligned}
D^{x x x x} & =D^{y y y y}=0 \\
D^{x x y y} & =\frac{1}{2}\left(D^{x x x x}-D^{x y x y}\right) \\
D^{x z x z}-D^{y z y z} & =-D_{a b}^{x x}+D_{a b}^{y y} \\
D^{x x z z}-D^{y y z z} & =2 D_{a b}^{x x}-2 D_{a b}^{y y}
\end{aligned}
$$

For all cases studied here, there exists an appropriate axes frame for which the fourth-rank tensor reduces to at most nine independent components when these relations are imposed instead of the 81 possible a priori.

\section{Results and discussion}

Geometrical deformations have been applied to the model complexes $\mathrm{O}\left[\mathrm{Ni}(\mathrm{NCH})_{4} \mathrm{CN}\right]_{2}(\mathrm{M} 1$, cases 1, 10, 11, 13 see figure 1) and $\mathrm{O}\left[\mathrm{Ni}(\mathrm{NCH})_{4} \mathrm{Cl}\right]_{2}(\mathrm{M} 2$, cases 2-9, and 12 see figure 2) to 
Table 1: Non-zero symmetric and antisymmetric components of the second-rank exchange tensor. ${ }^{45}$

\begin{tabular}{|c|c|c|c|c|}
\hline Point group ${ }^{\mathrm{a}}$ & $\kappa^{\mathrm{b}}$ & $\begin{array}{l}\text { Symmetric } \\
\text { components }\end{array}$ & $\begin{array}{l}\text { Antisymmetric } \\
\text { components }\end{array}$ & $\begin{array}{c}\text { Number of independent } \\
\text { components }\end{array}$ \\
\hline$C_{1}$ & +1 & $\mathrm{xx}, \mathrm{yy}, \mathrm{zz}, \mathrm{xy}, \mathrm{xz}, \mathrm{yz}$ & $\mathrm{xy}, \mathrm{xz}, \mathrm{yz}$ & 9 \\
\hline$C_{i}$ & -1 & $\mathrm{xx}, \mathrm{yy}, \mathrm{zz}, \mathrm{xy}, \mathrm{xz}, \mathrm{yz}$ & & 6 \\
\hline \multirow{2}{*}{$C_{S}^{\mathrm{c}}$} & -1 & \multirow{2}{*}{ xx,yy,zz,xy } & $\mathrm{xz}, \mathrm{yz}$ & 6 \\
\hline & +1 & & xy & 5 \\
\hline \multirow{2}{*}{$C_{2}$} & -1 & \multirow{2}{*}{ xx,yy,zz,xy } & $\mathrm{xz}, \mathrm{yz}$ & 6 \\
\hline & +1 & & xy & 5 \\
\hline$D_{2}$ & -1 & $\mathrm{xx}, \mathrm{yy}, \mathrm{zz}$ & xy & 4 \\
\hline \multirow{2}{*}{$C_{2 v}{ }^{\mathrm{d}}$} & -1 & \multirow[b]{2}{*}{ xx,yy,zz } & \multirow[t]{3}{*}{$\mathrm{xz}$} & 4 \\
\hline & +1 & & & 3 \\
\hline$C_{2 h}$ & -1 & xx,yy,zz,xy & & 4 \\
\hline$D_{2 h}$ & -1 & \multicolumn{2}{|l|}{$\mathrm{xx}, \mathrm{yy}, \mathrm{zz}$} & 3 \\
\hline$D_{2 d}$ & -1 & \multicolumn{2}{|l|}{$\mathrm{xx}=\mathrm{yy}, \mathrm{zz}$} & 2 \\
\hline$C_{n}$ & +1 & $\mathrm{xx}=\mathrm{yy}, \mathrm{zz}$ & xy & 3 \\
\hline$D_{n}$ & -1 & $\mathrm{xx}=\mathrm{yy}, \mathrm{zz}$ & xy & 3 \\
\hline$C_{n v}$ & +1 & \multicolumn{2}{|l|}{$\mathrm{xx}=\mathrm{yy}, \mathrm{zz}$} & 2 \\
\hline $\begin{array}{l}S_{n}, C_{n h} \\
D_{n h}, D_{n d}\end{array}$ & -1 & \multicolumn{2}{|l|}{$\mathrm{xx}=\mathrm{yy}, \mathrm{zz}$} & 2 \\
\hline
\end{tabular}

${ }^{\mathrm{a}}$ The $z$-axis is the highest-order rotation axis

${ }^{\mathrm{b}} \kappa=-1$ if the magnetic centers are exchanged by a symmetry operation and $\kappa=+1$ otherwise.

$\mathrm{c}$ The $\sigma_{h}$ plane is the $x y$ plane

$\mathrm{d}$ The two magnetic centers are in the $x z$ plane

Table 2: Non-zero symmetric components of the fourth-rank $\overline{\overline{\bar{D}}}_{a a b b}$ tensor.

\begin{tabular}{lcc} 
Point group $^{\mathrm{a}}$ & $\begin{array}{c}\text { Symmetric } \\
\text { components }\end{array}$ & $\begin{array}{c}\text { Number of } \\
\text { parameters }^{\mathrm{b}}\end{array}$ \\
\hline$C_{2}, C_{s}, C_{2 h}$ & $\begin{array}{c}\text { xxxx,yyyy,zzzz,xxyy,xxzz,yyzz } \\
\& \text { xyxy,xzxz,yyzz,xxxy,yyxy,zzxy }\end{array}$ & 14 \\
\hline$D_{2}, C_{2 v}, D_{2 h}$ & $\begin{array}{c}\text { xxxx,yyyy,zzzz,xxyy,xxzz,yyzz } \\
\& \text { xyxy,xzxz,yyzz }\end{array}$ & 9 \\
\hline$C_{n}, C_{n v}, C_{n h}, D_{n}, D_{n d}, D_{n h}$ & $\begin{array}{c}\text { xxxx=yyyy, zzzz, xxyy, xxzz=yyzz } \\
\& \text { xyxy, xzxz=yzyz }\end{array}$ & 6 \\
\hline $\begin{array}{l}\text { a The } z \text {-axis is the highest-order rotation axis } \\
\text { b Subject to reduction by additional relations (see text) }\end{array}$ &
\end{tabular}




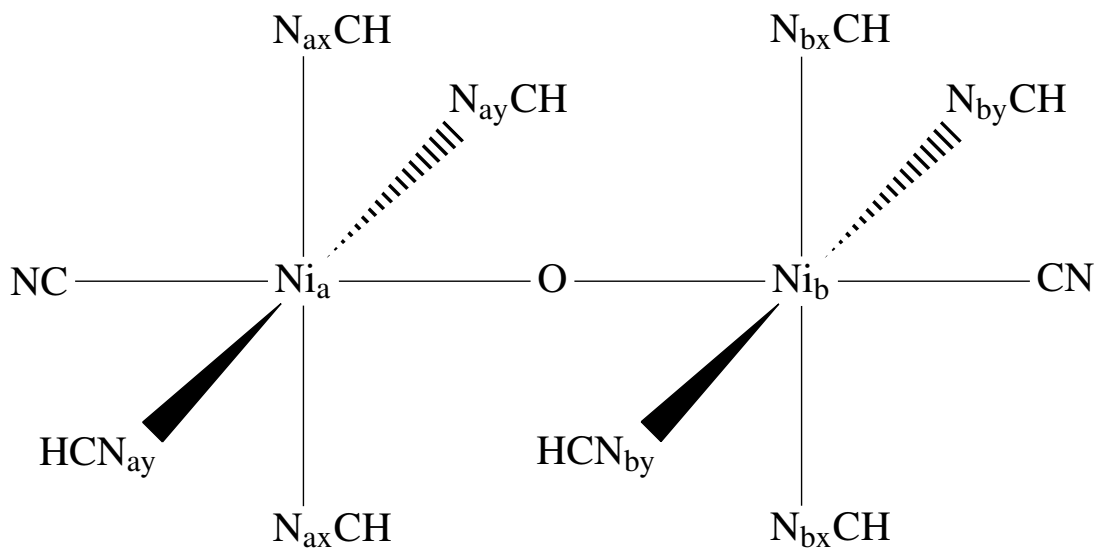

Figure 1: Scheme of the molecular model 1 (M1).

Geometrical parameters : distances $l_{a x}=\mathrm{Ni}_{a}-\mathrm{N}_{a x}, l_{a y}=\mathrm{Ni}_{a}-\mathrm{N}_{a y}, l_{b x}=\mathrm{Ni}_{b}-\mathrm{N}_{b x}, l_{b y}=\mathrm{Ni}_{b}-\mathrm{N}_{b y}$, angles $\theta_{a}=\left(\mathrm{N}_{a x}, \mathrm{Ni}_{a}, \mathrm{O}\right), \theta_{b}=\left(\mathrm{N}_{b x}, \mathrm{Ni}_{b}, \mathrm{O}\right)$ dihedral angles $\Phi_{x}=\left(\mathrm{N}_{a x}, \mathrm{Ni}_{a}, \mathrm{Ni}_{b}, \mathrm{~N}_{b x}\right), \Phi_{y}=\left(\mathrm{N}_{a y}, \mathrm{Ni}_{a}, \mathrm{Ni}_{b}, \mathrm{~N}_{b y}\right), \Phi_{x y}=\left(\mathrm{N}_{a x}, \mathrm{Ni}_{a}, \mathrm{Ni}_{b}, \mathrm{~N}_{b y}\right)$

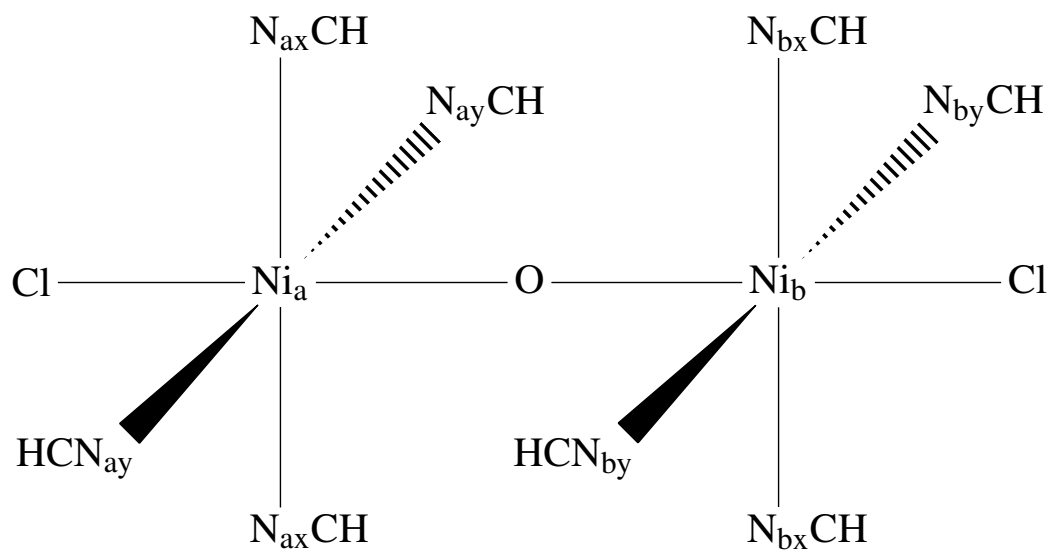

Figure 2: Scheme of the molecular model 2 (M2).

Geometrical parameters : distances $l_{a x}=\mathrm{Ni}_{a}-\mathrm{N}_{a x}, l_{a y}=\mathrm{Ni}_{a}-\mathrm{N}_{a y}, l_{b x}=\mathrm{Ni}_{b}-\mathrm{N}_{b x}, l_{b y}=\mathrm{Ni}_{b}-\mathrm{N}_{b y}$, dihedral angles $\Phi_{x}=\left(\mathrm{N}_{a x}, \mathrm{Ni}_{a}, \mathrm{Ni}_{b}, \mathrm{~N}_{b x}\right), \Phi_{y}=$ $\left(\mathrm{N}_{a y}, \mathrm{Ni}_{a}, \mathrm{Ni}_{b}, \mathrm{~N}_{b y}\right), \Phi_{x y}=\left(\mathrm{N}_{a x}, \mathrm{Ni}_{a}, \mathrm{Ni}_{b}, \mathrm{~N}_{b y}\right)$ 


(c)

Figure 3: Conventions used to schematize the nature of the magnetic anisotropy and to show the magnetic axes frame of the D-tensors. A prolate ellipsoid indicates an axial anisotropy while an oblate ellipsoid refers to a planar anisotropy. Hard axes and hard planes are represented in black while easy axes and easy planes are in red. In absence of rhombicity only one axis is represented.

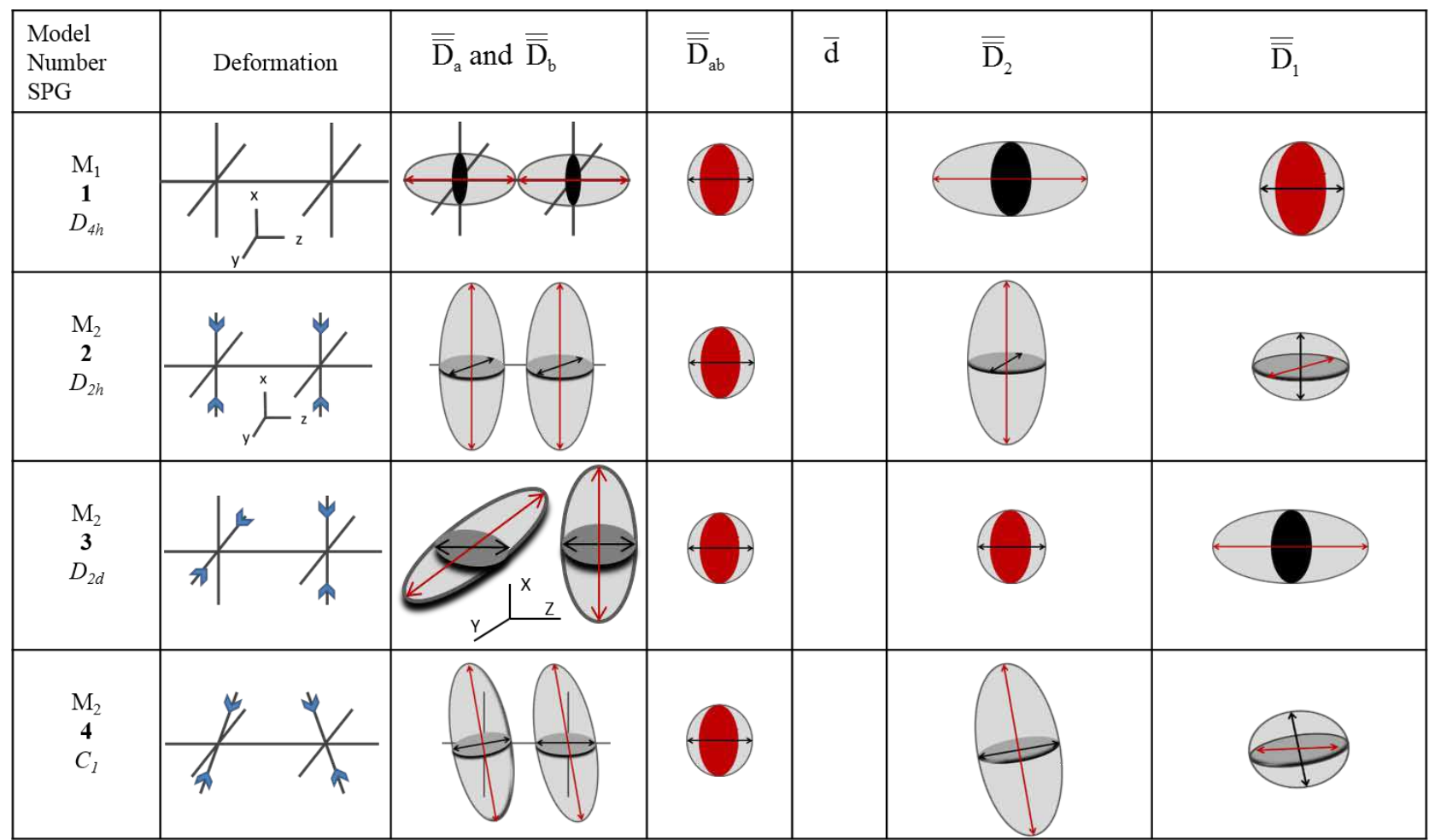

Figure 4: Ellipsoids representing the axial or planar anisotropy and magnetic anisotropy axes of the various local and molecular anisotropy tensors for different geometries in which local axial anisotropies have been imposed. The compound number and the model compound (either M1 or M2) are indicated in the left column. 


\begin{tabular}{|c|c|c|c|c|c|c|}
\hline $\begin{array}{l}\text { Model } \\
\text { Number } \\
\text { SPG }\end{array}$ & Deformation & $\overline{\overline{\mathrm{D}}}_{\mathrm{a}}$ and $\overline{\overline{\mathrm{D}}}_{\mathrm{b}}$ & $\overline{\overline{\mathrm{D}}}_{\mathrm{ab}}$ & $\overline{\mathrm{d}}$ & $\overline{\overline{\mathrm{D}}}_{2}$ & $\overline{\overline{\mathrm{D}}}_{1}$ \\
\hline $\begin{array}{c}\mathrm{M}_{2} \\
\mathbf{5} \\
D_{4 h}\end{array}$ & - & & & & & \\
\hline $\begin{array}{c}\mathrm{M}_{2} \\
\mathbf{6} \\
D_{2 h}\end{array}$ & 舟 & & & & & \\
\hline $\begin{array}{c}\mathrm{M}_{2} \\
7 \\
D_{2 d}\end{array}$ & 4 & & & & & \\
\hline $\begin{array}{c}\mathrm{M}_{2} \\
\mathbf{8} \\
D_{2}\end{array}$ & 斥 & & & $\longleftarrow$ & & \\
\hline $\begin{array}{c}\mathrm{M}_{2} \\
\mathbf{9} \\
C_{2 v}\end{array}$ & $\frac{\pi}{\pi}$ & & & & & \\
\hline $\begin{array}{c}\mathrm{M}_{1} \\
\mathbf{1 0} \\
D_{2 d}\end{array}$ & 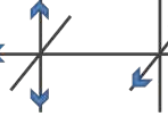 & & & & & \\
\hline
\end{tabular}

Figure 5: Ellipsoids representing the axial or planar anisotropy and magnetic anisotropy axes of the various local and molecular anisotropy tensors for different geometries in which local planar anisotropies have been imposed. The compound number and the model compound (either M1 or M2) are indicated in the left column. 


\begin{tabular}{|c|c|c|c|c|c|c|}
\hline $\begin{array}{l}\text { Model } \\
\text { Number } \\
\text { SPG }\end{array}$ & Deformation & $\overline{\mathrm{D}}_{\mathrm{a}}$ and $\overline{\mathrm{D}}_{\mathrm{b}}$ & $\overline{\overline{\mathrm{D}}}_{\mathrm{ab}}$ & $\overline{\mathrm{d}}$ & $\overline{\overline{\mathrm{D}}}_{2}$ & $\overline{\overline{\mathrm{D}}}_{1}$ \\
\hline $\begin{array}{l}\mathrm{M}_{1} \\
\mathbf{1 1} \\
C_{4 v}\end{array}$ & & & & & & \\
\hline $\begin{array}{l}\mathrm{M}_{1} \\
\mathbf{1 2} \\
C_{2 v}\end{array}$ & & & & & & \\
\hline $\begin{array}{l}\mathrm{M}_{2} \\
\mathbf{1 3} \\
C_{2 v}\end{array}$ & 7 & & & & & \\
\hline
\end{tabular}

Figure 6: Ellipsoids representing the axial or planar anisotropy and magnetic anisotropy axes of the various local and molecular anisotropy tensors for different geometries in which both an axial and a planar local anisotropies have been imposed. The compound number and the model compound (either M1 or M2) are indicated in the left column.

tune the characteristics of the local and molecular anisotropies (see figures 3, 4, 5 and table 4). The stronger field exerted by the $\mathrm{CN}^{-}$ligand makes it possible to study some extra combinations of local anisotropies that are not easily realized in the other complex, as specified below. Both model complexes show strong antiferromagnetic isotropic coupling and have an $S=0$ ground state.

For each structure, the parameters of three different models were extracted: (i) The local ZFS parameters $D_{a}^{l o c}, E_{a}^{l o c}, D_{b}^{l o c}, E_{b}^{l o c}$; (ii) the giant-spin Hamiltonian parameters $D_{2}, E_{2}$ for the quintet and $D_{1}, E_{1}$ for the triplet spin manifolds; and (iii) all the parameters of the multispin Hamiltonian. The values are listed in table 3 . The proper magnetic axes of all the tensors have been extracted and are represented in figures 4 (both centers with axial local anisotropy), 5 (both centers with planar local anisotropy) and 6 (for axial and planar local anisotropies). These figures report pictures of the applied deformations and of the resulting anisotropy ellipsoids that provide a visualization of the nature of the magnetic anisotropies (both local and molecular for the quintet and triplet states); the direction of the Dzyaloshinskii Moriya vector is also indicated when it is not zero. Note that the conventions used to represent the anisotropy in figures 4, 5 and 6 are given in figure 3 . 
Compression, stretching and angular distortion generate local anisotropies with peculiar and different features. The $D_{4 h}$ geometry $(5)$ of $\mathrm{O}\left[\mathrm{Ni}(\mathrm{NCH})_{4} \mathrm{Cl}\right]_{2}$ is such that $\mathrm{Z}$ is a hard axis of magnetization for both centers and they do not exhibit any local rhombicity. In the $D_{2 h}$ structures (6 and 2) the bonds have been stretched or compressed in a single direction to generate either two parallel easy planes (table 5) or two collinear easy axes (table 4) of magnetization. In the $D_{2 d}$ structures (7 and $\mathbf{3}$ ), the deformations are applied to different axes (X and $\mathrm{Y}$ ) such that the easy planes or the easy axes are orthogonal. Angular distortions (of 10 degrees on each sites) have been applied to both $D_{2 h}$ geometries to generate the $D_{2}$ structure in which the local easy planes $(\mathbf{8})$ are no longer parallel. The $C_{2 v}(\mathbf{9})$ geometry illustrates the cases of two local planar anisotropies, where only one of them is rhombic. In the $C_{2 v}(\mathbf{1 2})$ geometry, one center has a planar anisotropy while the other has an axial anisotropy and the easy axis is parallel to the easy plane. Finally, in the $C_{1}$ structure (4) the two local anisotropies are axial; one of the easy axes is in the (XY) plane and the other in the (XZ) plane.

The ligand $\mathrm{CN}^{-}$produces a stronger ligand field which permits to study other combinations of local anisotropies. In the $D_{4 h}$ structure $(\mathbf{1})$ of $\mathrm{O}\left[\mathrm{Ni}(\mathrm{NCH})_{4} \mathrm{CN}\right]_{2}$, the local axial anisotropies share the same easy axis $\mathrm{Z}$. The $C_{4 v}$ structure (11) has one axial local anisotropy with the easy axis $\mathrm{Z}$ and a local planar anisotropy with the easy plane (XY). In the $C_{2 v}$ structure (13), one center has an axial anisotropy with the easy axis $\mathrm{Z}$ while the other has a planar anisotropy with the easy plane (YZ).

From the comparison of the values of the different local and molecular anisotropy parameters, a series of conclusions can be extracted, which will be discussed below in a point-by-point fashion.

- The magnetic axes frames and local anisotropy parameters extracted from the calculations performed with one or two active magnetic centers are very similar, showing the transferability of these parameters from the "embedded" monomer to the dimer. The small discrepancies are due to the bias introduced in the calculations of the local tensors by the arbitrarily imposed closed-shell character of the inactive $\mathrm{Ni}(\mathrm{II})$ center. The values of the local anisotropy parameters that should be considered as the most precise are those extracted from the calcu- 
Table 3: Values of extracted parameters in $\mathrm{cm}^{-1}$. The values of the local tensors components marked with a superindex "loc" have been extracted from calculations performed with a single magnetic active center. Note that ' 0 ' is used for terms that are strictly zero for symmetry reasons, while ' 0.0 ' indicates that the extracted value of the parameter is smaller than $0.05 \mathrm{~cm}^{-1}$.

\begin{tabular}{|c|c|c|c|c|c|c|c|c|c|c|c|c|c|}
\hline Cases & $\mathbf{1}$ & $\mathbf{2}$ & $\mathbf{3}$ & $\mathbf{4}$ & $\mathbf{5}$ & $\mathbf{6}$ & $\mathbf{7}$ & $\mathbf{8}$ & $\mathbf{9}$ & $\mathbf{1 0}$ & $\mathbf{1 1}$ & $\mathbf{1 2}$ & $\mathbf{1 3}$ \\
$\mathrm{SPG}$ & $D_{4 h}$ & $D_{2 h}$ & $D_{2 d}$ & $C_{1}$ & $D_{4 h}$ & $D_{2 h}$ & $D_{2 d}$ & $D_{2}$ & $C_{2 v}$ & $D_{2 d}$ & $C_{4 v}$ & $C_{2 v}$ & $C_{2 v}$ \\
\hline$D_{a}^{l o c}$ & -3.1 & -21.9 & -19.4 & -22.5 & 10.0 & 9.9 & 10.0 & 8.5 & 9.9 & 15.0 & -4.3 & 2.2 & -3.8 \\
\hline$D_{b}^{l o c}$ & -3.1 & -21.9 & -19.4 & -24.4 & 10.0 & 9.9 & 10.0 & 8.5 & 9.6 & 15.0 & 2.1 & -21.7 & 5.4 \\
\hline$E_{a}^{l o c}$ & 0 & 3.7 & 2.8 & 0.6 & 0 & 1.6 & 1.5 & 1.8 & 1.5 & 1.3 & 0 & 0.1 & 1.1 \\
\hline$E_{b}^{l o c}$ & 0 & 3.7 & 2.8 & 0.1 & 0 & 1.6 & 1.5 & 1.8 & 0.0 & 1.3 & 0 & 2.6 & 0.0 \\
\hline$D_{2}$ & -2.4 & -6.7 & 2.5 & -6.6 & 2.5 & 2.5 & 2.5 & 1.6 & 2.5 & -2.2 & -1.5 & -3.6 & -2.5 \\
$E_{2}$ & 0 & 0.7 & 0 & 0.6 & 0 & 0.5 & 0 & 0.2 & 0.2 & 0 & 0 & 0.2 & 0.3 \\
\hline$D_{1}$ & 7.6 & 19.2 & -6.9 & 18.6 & -6.6 & -6.7 & -6.6 & -3.7 & -6.7 & 9.7 & 5.2 & 8.6 & 8.0 \\
$E_{1}$ & 0 & 2.3 & 0 & 2.4 & 0 & 1.4 & 0 & 0.5 & 0.7 & 0 & 0 & 1.4 & 0.9 \\
\hline$J$ & 38.9 & 50.3 & 49.7 & 49.8 & 40.9 & 41.1 & 41.1 & 41.0 & 41.8 & 30.6 & 40.3 & 38.6 & 37.2 \\
\hline$D_{a}$ & -2.2 & -21.1 & -21.9 & -20.7 & 10.9 & 12.0 & 12.1 & 9.3 & 12.0 & 16.6 & -3.6 & 4.6 & -2.1 \\
$D_{b}$ & -2.2 & -21.1 & -21.9 & -21.3 & 10.9 & 12.0 & 12.1 & 9.3 & 12.0 & 16.6 & 1.1 & -20.3 & 4.2 \\
\hline$E_{a}$ & 0 & 0.4 & 0.3 & 0.6 & 0 & 1.4 & 1.4 & 1.4 & 1.4 & 3.1 & 0 & 0.0 & 0.0 \\
$E_{b}$ & 0 & 0.4 & 0.3 & 0.1 & 0 & 1.4 & 1.4 & 1.4 & 0.0 & 3.1 & 0 & 0.9 & 0.5 \\
\hline$D_{a b}$ & 0.3 & 0.3 & 0.3 & 0.3 & 0.4 & 5.2 & 0.4 & 0.4 & 5.1 & -4.7 & 0.3 & 0.3 & -4.7 \\
$E_{a b}$ & 0 & 0.0 & 0 & 0.0 & 0 & 0.9 & 0 & 0.0 & 0.5 & 0 & 0 & 0.0 & 0.6 \\
\hline$D_{z z z z}$ & -4.9 & -4.0 & -5.4 & -3.6 & -3.8 & -4.8 & -4.9 & -4.9 & -4.9 & 5.5 & -3.6 & -3.0 & -5.4 \\
\hline$D_{x x z z}$ & 0 & 0 & 0 & 0 & 0 & -1.3 & 0 & 0 & -2.2 & 3.3 & 0 & 0 & 2.2 \\
$D_{y y z z}$ & 0 & 0 & 0 & 0 & 0 & -5.0 & 0 & 0 & -4.1 & 3.3 & 0 & 0 & 4.7 \\
\hline$D_{x x y y}$ & 2.5 & 2.0 & 2.7 & 1.8 & 1.9 & 8.7 & 2.4 & 2.4 & 8.7 & -9.4 & 1.8 & 1.5 & -4.2 \\
\hline$D_{x y x y}$ & -1.2 & -1.0 & -1.4 & -0.9 & -0.9 & -4.4 & -1.2 & -1.2 & 4.4 & 4.7 & -0.9 & -0.7 & 2.1 \\
\hline$D_{x z x z}$ & -1.3 & -1.1 & -1.4 & -1.3 & 0 & -0.6 & -1.2 & -1.3 & -0.1 & -0.3 & -0.9 & -0.8 & -2.4 \\
$D_{y z y z}$ & -1.3 & -1.0 & -1.4 & -1.3 & 0 & 1.3 & -1.2 & -1.3 & 0.8 & -0.3 & -0.9 & -0.8 & -3.6 \\
\hline
\end{tabular}


lations in which the two magnetic centers are active.

- The values of $D_{a b}$ and $E_{a b}$ (anisotropic exchange parameters) are very small and slightly more important when the local anisotropy is planar than for axial local anisotropy. The anisotropy of the interaction between the two anisotropic centers originates essentially in the fourth-rank tensor components. The planar nature of the anisotropy caused by $\overline{\bar{D}}_{a b}$ appears to be almost unaffected by the distortions in the considered systems, except for cases $\mathbf{1 0}$ and 13.

- The giant-spin second-rank tensors $\overline{\bar{D}}_{1}$ and $\overline{\bar{D}}_{2}$ exhibit opposite anisotropic characteristics, the axes and planes of easy and hard magnetization are systematically opposite. For simplicity, only the values and features of $\overline{\bar{D}}_{2}$ will be commented in the subsequent points. One may also note that the absolute values of the anisotropy parameters are larger in the triplet $\left(\overline{\bar{D}}_{1}\right)$ than in the quintet $\left(\overline{\bar{D}}_{2}\right)$, as already explained by Boča. ${ }^{22}$ Due to the presence of the fourth-rank tensor in the model Hamiltonian considered in this work, and in some cases due to the mismatch between the local tensor anisotropy axes, the relations between the tensors found in this study are different than those proposed earlier. ${ }^{22}$

- As expected, stretching and compression induce opposite local anisotropy behavior with planar and axial anisotropy, respectively. This results in positive and negative $D_{2}$ values.

- The Dzyaloshinskii-Moriya vector is either very small or strictly zero by symmetry. Among the considered cases, non-zero values were only found for the $C_{2 v}$ and $C_{1}$ symmetry point groups. Since the obtained values were lower than $0.05 \mathrm{~cm}^{-1}$, they have not been reported here.

- When both local anisotropies are axial (see figure 4), the nature of the anisotropy of the quintet is usually axial except when the two local axes are orthogonal (case 3). The easy axis always bisects the two local easy axes (and is therefore parallel or collinear -i.e. parallel and having a common point- with the local axes when these ones are already parallel or 
collinear). One should note that the values of the molecular anisotropy parameters are usually drastically reduced in comparison to the local ones. The most interesting situation (case 1) occurs when the local axes are collinear, as expected. One should note that in such a case the absence of local rhombicity is maintained in the molecular magnetic anisotropy of the complex and that there is a (small) synergistic effect: the overall magnetic axial parameter value for the high-spin block is $-2.4 \mathrm{~cm}^{-1}$ while the local ones are $-2.2 \mathrm{~cm}^{-1}$.

- When both local anisotropies are planar (see figure 5), the molecular magnetic anisotropy is usually planar and here again the values of the anisotropic parameters are smaller than the local ones. Introducing local rhombicity may actually be interesting for tuning the nature of the overall anisotropy of the complex. Indeed, if the local planes are parallel and the local easy axes are perpendicular, the overall magnetic anisotropy does not exhibit any rhombicity (case 7). When the local easy axes have an angle, the resulting easy axis bisects the two local ones (cases 6 and 8). The most interesting situation occurs when the local easy planes are orthogonal and the local easy axes are collinear, since it is possible to generate a purely axial anisotropy (negative axial parameter and no rhombicity).

- When the nature of the local anisotropies is different (one axial and one planar, cases 11-13 see figure 6), it is possible to generate an axial molecular magnetic anisotropy independent of the orientation of the local easy axis and plane (cases 11 and 12). Two interesting situations should be noted, i) the absence of local rhombicities (case 11) leads to the absence of rhombic molecular magnetic anisotropy, and ii) a synergistic effect occurs when the local easy axes are collinear as in case 13 , where the local axial parameter $D_{a}$ is $-2.1 \mathrm{~cm}^{-1}$ while the overall axial parameter $D_{2}$ is $-2.5 \mathrm{~cm}^{-1}$.

\section{Summary and perspectives}

This work is a follow-up of previous theoretical studies in which it was shown that both the commonly applied giant-spin and multispin Hamiltonians are not appropriate for the description of 
the anisotropy of binuclear complexes, in particular in the weak-exchange limit. ${ }^{18,19}$ Because the values of the numerous parameters of the multispin Hamiltonian involving the fourth-rank tensor components could not be extracted up to now, ${ }^{18}$ a method of extraction that makes use of the effective Hamiltonian theory has been implemented here and successfully applied to complexes exhibiting different local anisotropies. Rules to predict vanishing values of symmetric and antisymmetric second-rank exchange tensors and symmetric fourth-rank symmetric tensors were presented, illustrated (see figures 4, 5 and 6) and tested on model molecules.

Several conclusions can be drawn from this work. Among the most important ones, one should mention that the anisotropy of the interactions between the two magnetic centers is essentially caused by the symmetric fourth-rank tensor while the second-rank exchange tensor components are almost always negligible. As a consequence, the relations between the molecular anisotropy of the complex and the local ones are no more quantitatively valid. Nevertheless, from a qualitative point of view the nature of the overall magnetic anisotropy can be anticipated from the local anisotropy, except when the complex belongs to the weak-exchange regime. One should also note that the Dzyaloshinskii-Moriya term is very small in the considered cases and that non zero values were only obtained for symmetry point groups lower than $\mathrm{C}_{2 v}$. Obtaining larger antisymmetric terms would require other deformations, such as changing the $\left(\mathrm{Ni}_{a}, \mathrm{O}, \mathrm{Ni}_{b}\right)$ angle away from $180^{\circ}$.

Combinations of local anisotropies were found to show synergistic effects (increased axiality of the molecular magnetic anisotropy in comparison to the local ones) in three cases. The first one occurs when two local axial anisotropies with collinear local easy axes are combined. The molecular axial anisotropy is larger than the sum of the local anisotropies. This is in line with the results of experimentalists working in the domain of polynuclear single molecule or single chain magnets for instance. ${ }^{46}$ The second case of synergy is observed when two local planar, rhombic anisotropies are combined, leading to a purely axial (no rhombicity) molecular anisotropy when the local easy axes are collinear and the local easy planes are perpendicular. Finally, axial and planar local anisotropies may lead to an axial molecular anisotropy and a synergy occurs when the local easy axis of one center is in the local easy plane of the other center. 
To the best of our knowledge, values of the multispin parameters are extremely difficult to extract from experiment. In this respect, a theoretical approach that provides both qualitative and quantitative information concerning magnetic anisotropy appears as an interesting mean to help synthetic chemists to control and improve this property. Forthcoming papers will be devoted to the application of the here-presented method of extraction to existing, synthesized compounds.

\section{Acknowledgment}

Financial support has been provided by the Spanish administration (Project CTQ2011-23140), and the Generalitat de Catalunya (Project 2009SGR462 and Xarxa d'R+D+I en Química Teòrica $i$ Computacional, XRQTC). This work was supported by the French Centre National de la Recherche Scientifique (CNRS), by the Université de Toulouse and by the Agence Nationale de la Recherche (ANR) (Project TEMAMA ANR-09-BLAN-0195-01).

\section{References}

(1) Sessoli, R.; Tsai, H. L.; Schake, A. R.; Wang, S.; Vincent, J. B.; Folting, K.; Gatteschi, D.; Christou, G.; Hendrickson, D. N. J. Am. Chem. Soc. 1993, 115, 1804-1816.

(2) Sessoli, R.; Gatteschi, D.; Caneschi, M. A., A. Novak Nature 1993, 365, 141-143.

(3) Gatteschi, D.; Sessoli, R.; Villain, J. Molecular Nanomagnets; Oxford University Press, 2006.

(4) Milios, C. J.; Vinslava, A.; Wernsdorfer, W.; Moggach, S.; Parsons, S.; Perlepes, S. P.; Christou, G.; Brechin, E. K. J. Am. Chem. Soc. 2007, 129, 2754-2755.

(5) Yoshihara, D.; Karasawa, S.; Koga, N. J. Am. Chem. Soc. 2008, 130, 10460-10461.

(6) Mannini, M.; Pineider, F.; Sainctavit, P.; Danieli, C.; Otero, E.; Sciancalepore, C.; Talarico, A.; Arrio, M.-A.; Cornia, A.; Gatteschi, D.; Sessoli, R. Nat Mater. 2009, 8, 194-197.

(7) Leuenberger, M. N.; Loss, D. Nature 2001, 410, 789-793. 
(8) Ardavan, A.; Rival, O.; Morton, J. J. L.; Blundell, S. J.; Tyryshkin, A. M.; Timco, G. A.; Winpenny, R. E. P. Phys. Rev. Lett. 2007, 98, 057201.

(9) Stamp, P. C. E.; Gaita-Arino, A. J. Mater. Chem. 2009, 19, 1718-1730.

(10) Chibotaru, L. F.; Ungur, L. J. Chem. Phys. 2012, 137, 064112.

(11) Zadrozny, J. M.; Liu, J.; Piro, N. A.; Chang, C. J.; Hill, S.; Long, J. R. Chem. Commun. 2012, 48, 3927-3929.

(12) Cirera, J.; Ruiz, E.; Alvarez, S.; Neese, F.; Kortus, J. Chem. Eur. J. 2009, 15, 4078-4087.

(13) Maurice, R.; de Graaf, C.; Guihéry, N. J. Chem. Phys. 2010, 133, 084307.

(14) Atanasov, M.; Ganyushin, D.; Pantazis, D. A.; Sivalingam, K.; Neese, F. Inorg. Chem. 2011, $50,7460-7477$.

(15) McGarvey, B.; Telser, J. Inorg. Chem. 2012, 51, 6000-6010.

(16) Ruamps, R.; Maurice, R.; Batchelor, L. J.; Boggio-Pasqua, M.; Guillot, R.; Barra, A.-L.; Liu, J.; Bendeif, E.-E.; Pillet, S.; Hill, S.; Mallah, T.; Guihéry, N. J. Am. Chem. Soc. 2013, 135, 3017-3026.

(17) Gomez-Coca, S.; Cremades, E.; Aliaga-Alcalde, N.; Ruiz, E. J. Am. Chem. Soc 2013, 135, $7010-7018$.

(18) Maurice, R.; Guihéry, N.; Bastardis, R.; de Graaf, C. J. Chem. Theory Comput. 2010, 6, $55-65$.

(19) Maurice, R.; de Graaf, C.; Guihéry, N. Phys. Rev. B 2010, 81, 214427.

(20) Kahn, O. Molecular Magnetism; VCH Publishers, 1993.

(21) Boča, R. Theoretical foundations of molecular magnetism; Elsevier: Amsterdam, 1999.

(22) Boča, R. Coord. Chem. Rev. 2004, 248, 757-815. 
(23) Boča, R.; Herchel, R. Coord. Chem. Rev. 2010, 254, 2973-3025.

(24) Calzado, C. J.; Cabrero, J.; Malrieu, J.-P.; Caballol, R. J. Chem. Phys. 2002, 116, 2728-2747.

(25) Calzado, C. J.; Cabrero, J.; Malrieu, J.-P.; Caballol, R. J. Chem. Phys. 2002, 116, 3985-4000.

(26) Bastardis, R.; Guihéry, N.; de Graaf, C. J. Chem. Phys. 2008, 129, 104102.

(27) Malrieu, J.-P.; Caballol, R.; Calzado, C. J.; de Graaf, C.; Guihéry, N. Chem. Rev. 2014, 114, 429-492.

(28) Maurice, R.; Bastardis, R.; de Graaf, C.; Suaud, N.; Mallah, T.; Guihéry, N. J. Chem. Theory Comput. 2009, 5, 2977-2984.

(29) Maurice, R.; Pradipto, A. M.; Guihéry, N.; Broer, R.; de Graaf, C. J. Chem. Theory Comput. 2010, 6, 3092-3101.

(30) Ruamps, R.; Batchelor, L. J.; Maurice, R.; Gogoi, N.; Jiménez-Lozano, P.; Guihéry, N.; de Graaf, C.; Barra, A.-L.; Sutter, J.-P.; Mallah, T. Chem. Eur. J. 2013, 19, 950-956.

(31) Maurice, R.; Verma, P.; Zadrozny, J. M.; Luo, S.; Borycz, J.; Long, J. R.; Truhlar, D. G.; Gagliardi, L. Inorg. Chem. 2013, 52, 9379-9389.

(32) Bloch, C. Nucl. Phys. 1958, 6, 329-347.

(33) des Cloizeaux, J. Nucl. Phys. 1960, 20, 321-346.

(34) Malmqvist, P.-Å.; Roos, B. O.; Schimmelpfennig, B. Chem. Phys. Lett. 2002, 357, 230-240.

(35) Roos, B. O.; Malmqvist, P.-Å. Phys. Chem. Chem. Phys. 2004, 6, 2919-2927.

(36) Karlström, G.; Lindh, R.; Malmqvist, P.-Å.; Roos, B. O.; Ryde, U.; Veryazov, V.; Widmark, P.-O.; Cossi, M.; Schimmelpfennig, B.; Neogrady, P.; Seijo, L. Comput. Mater. Sci. 2003, 28, 222-239. 
(37) Aquilante, F.; De Vico, L.; Ferré, N.; Ghigo, G.; Malmqvist, P.-A.; Neogrády, P.; Pedersen, T. B.; Pitonák, M.; Reiher, M.; Roos, B. O.; Serrano-Andrés, L.; Urban, M.; Veryazov, V.; Lindh, R. J. Comp. Chem. 2010, 31, 224-247.

(38) Roos, B. O.; Lindh, R.; Malmqvist, P.-Å; Veryazov, V.; Widmark, P.-O. J. Phys. Chem. A 2004, 108, 2851-2858.

(39) Roos, B. O.; Lindh, R.; Malmqvist, P.-Å; Veryazov, V.; Widmark, P.-O. J. Phys. Chem. A 2005, 109, 6575-6579.

(40) Gatteschi, D.; Sessoli, R. Angew. Chem. Int. Ed. 2003, 42, 268-297.

(41) Barra, A. L.; Gatteschi, D.; ; Sessoli, R. Phys. Rev. B 1997, 56, 8192-8198.

(42) Hill, S.; Perenboom, J. A. A. J.; Dalal, N. S.; Hathaway, T.; Stalcup, T.; Brooks, J. S. Phys. Rev. Lett. 1998, 80, 2453-2456.

(43) Wilson, A.; Lawrence, J.; Yang, E.-C.; Nakano, M.; Hendrickson, D. N.; Hill, S. Phys. Rev. $B$ 2006, 74, 140403.

(44) Abragam, A.; Bleaney, B. Electron paramagnetic resonance of transition ions; Dover Publications: Dover, New York, 1986.

(45) Buckingham, A. D.; Pyykko, P.; Robert, J. B.; Wiesenfeld, L. Mol. Phys. 1982, 46, 177-182.

(46) Gogoi, N.; Thlijeni, M.; Duhayon, C.; Sutter, J.-P. Inorg. Chem. 2013, 52, 2283-2285. 


\section{Table of contents:}

\section{Artwork:}
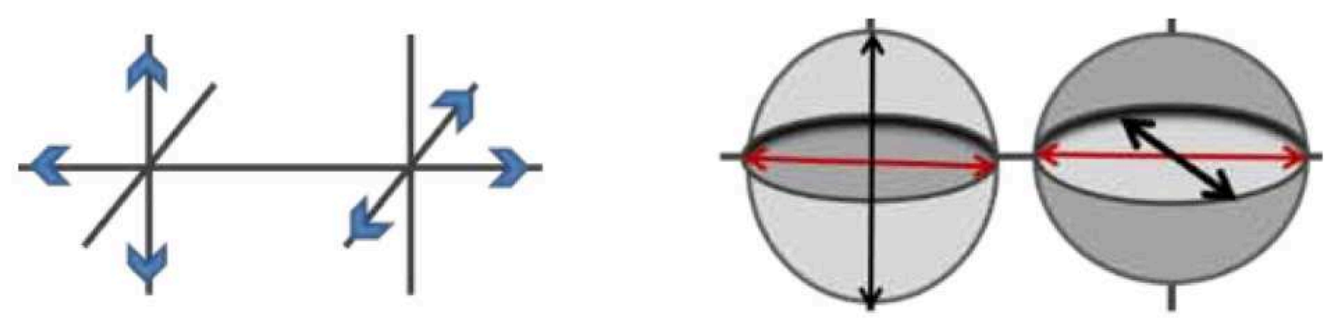

\section{Local LF distortions leading to local planar anisotropies}

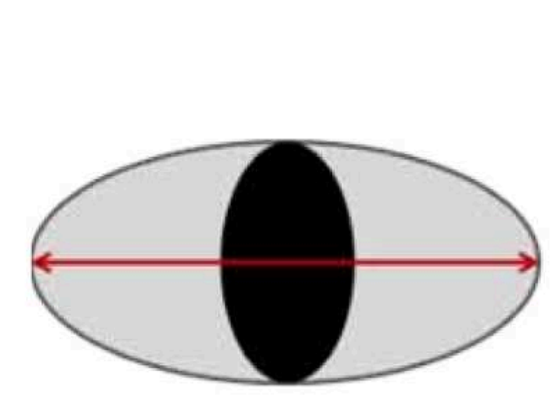

$\downarrow$

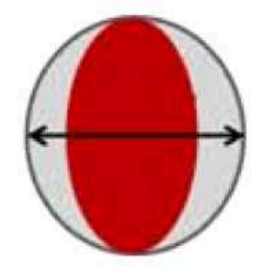

\section{Purely axial $S=2$ anisotropy Purely planar $S=1$ anisotropy}

\section{Synopsis:}

The role of local distortions on the molecular magnetic anisotropies of model binuclear $\mathrm{Ni}(\mathrm{II}) \mathrm{com}$ plexes is assessed with wave function based methods. The anisotropy parameters of the multispin and giant-spin model Hamiltonians are unambiguously extracted with the effective Hamiltonian theory. It is shown that the combination of some local distortions can lead to constructive or destructive interactions between the local anisotropies, which can be used to tune the magnetic anisotropy in polynuclear complexes. 